SUPERCONDUCTIVTTY, CRYOGENICS, AND VACUUM TECHNOLOGY FOR LINEAR ACCELERATORS*

Edward L. Garwin

Stanford Linear Accelerator Center, Stanford University, Stanford, California 94305

\begin{abstract}
The conventional, $10^{-8}$ Torr-range, ion-pumped vacuum system of the SLAC 3.2-km linear accelerator is briefly described, along with typical performance data. Since polarized electron sources are of increasing importance to high-energy research, the necessary physical environments for such sources is given, and differential pumping and/or isolation systems capable of maintaining those conditions while protecting both source and accelerator are discussed. The development of microwave superconducting linear accelerators is summarized. Emphasis is on vacuum and materials processing techniques necessary to the maintenance of low surface resistance and high electric and magnetic fields. Problems remaining and prospects for their solution are briefly described, along with details of the physical processes taking place at the vacuum-metal interface.
\end{abstract}

\footnotetext{
*Work supported by the U.S. Atomic Energy Commission.
} 


\section{Accelerator Vacuum System}

The vacuum system for the 3.2-km Stanford Lincar Accelerator has been described in detail elsewhere, ${ }^{1,2)}$ so only a brief summary will be given here. The cross-section of the linear accelerator housing and klystron gallery is shown in Fig. 1. The gauges, pumps, and all valves except for in-line valves isolating one 102-meter accelerator sector from another arc located above ground in the klystron gallery. The copper disc-loaded waveguide (shown in Fig. 2) which constitutes the accelerator, and the 12.2-m support and alignment girder assembly (shown in Fig. 3) are located below-ground to allow for radiation shielding. Fig. 4 is a view looking east along the accelerator tunnel, showing the completed installation. The all-metal, high-vacuum system which maintains the $20-\mathrm{cm}$ sector manifold at a pressure of $1.5 \times 10^{-8}$ Torr during machine operation $\left(7.5 \times 10^{-9}\right.$ Torr with the accelerating field off) is shown schematically in Fig. 5. One such system is provided for each of the thirty 102-m sectors comprising the machine. Four 500 liter/sec diode getter-ion pumps located in the klystron gallery evacuate the accelerator and waveguides through interconnecting stainless-steel manifolds. A pump can be removed for servicing without interference with accelerator operations by closing the associated $15-\mathrm{cm}$ valve. Similarly, an individual klystron can be replaced by closing the $8-\mathrm{cm}$ valve connecting it to the pumping manifold and the waveguide

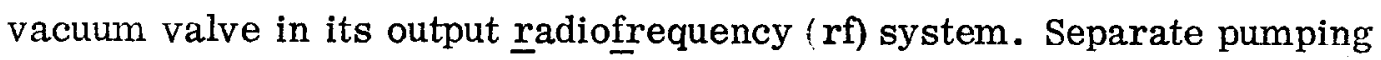
systems are provided for rough-pumping the accelerator, for the $61-\mathrm{cm}$ 
alignment light-pipe, and for the beam switchyard.

\section{Polarized Electron Sources}

Certain high-energy physics experiments require a source of Iongitudinally polarized electrons, whose polarization is known, and which can be reversed upon demand. There are four fairly well-developed methods for producing such electrons:

1) Photoionization (using unpolarized light) of a $\mathrm{Li}^{6}$ atomic beam which has been state-selected by a sextupole magnet (PEGGY source).

2) Photoionization using a circularly polarized, frequency doubled dye laser beam incident on Cs vapor (Fano effect source).

3) Photoemission (using unpolarized light) from ferromagnetic materials (EuO, or $\mathrm{Fe}$ ) held in longitudinal magnetic fields at temperatures below the Curie point $\left(69^{\circ} \mathrm{K}\right.$ for $\left.\mathrm{EuO}\right)$.

4) Field emission from $W$ needles, coated with EuS and held in a longitudinal magnetic field at a temperature below the Curie point.

The first two types of source have, in the $10^{-7}$ Torr source region, large quantities of hot alkali metals. Should these find their way into the injector section of the accelerator, the work function of the surfaces is lowered to about $2.5 \mathrm{eV}$, and the normally present field emission from the section has been calculated to increase by up to a factor $10^{6}$, and this has been verified by d.c. measurements. In order to prevent such an accident, the arrangement shown in Fig. 6 is being installed with the $\mathrm{Li}^{6}$ source designated as PEGGY, presently under construction at Yale University for use at SLAC.

The rationale is one of protection of the accelerator. Under normal, highvacuum conditions, the refrigerated copper surface traps lithium metal, and effectively prevents its entrance into the accelerator. ${ }^{3)}$ The most dangerous conditions are those in which the PEGGY source is vented to atmospheric 
pressure either inadvertently, or by catastrophic failure of the quartz window which transmits the ultraviolet radiation used to photoionize the lithium. Under such circumstances, the lithium would be carried into the injector, irrespective of any baffling scheme. Therefore, when the two air-operated valves of Fig. 6 are open, a signal from any one of the six ion gauges will close all three of the fast valves within $10 \mathrm{msec}$. When the two air-operated valves are closed, signals from only the two ion gauges on the PEGGY source are rendered ineffective for closing the fast valves. A lithium detector (operating on the principle of measuring field emission) will be installed between two of the fast valves, and after the valves have been triggered closed, only in the proven absence of lithium will the valve at the injector section be allowed to open.

For the solid-state sources listed as 3-4 above, no free alkali-metals are present, but in order to preserve polarized photoemission, pressures of $10^{-10}$ to $10^{-11}$ Torr are required. Differential pumping schemes are required to produce these pressures at the source, with pressures of $10^{-8}$ Torr at the accelerator. Fortunately, since the sources require both low temperatures and magnetic fields, it is expeditious to use superconducting magnets, and obtain differential pumping by using liquid-helium-cooled shrouds as cryopumps. (The neon, hydrogen and helium which are not cryogenically pumped do not usually have a deleterious effect on the photoemitting surfaces.)

3. Superconducting Linear Accelerators

\subsection{Introduction}

Twelve years ago, measurements on lead-plated, S-band cavities at the High Energy Physics Laboratory of Stanford University (HEPL) demonstrated the feasibility of superconducting linear accelerators (SCA) by producing $Q^{\prime} s$ of $10^{8}-10^{9}$, and peak rf magnetic fields in the range of $100 \mathrm{G}$. In the years 
immediately following, further improvements in rf cavity properties were achieved, affirming the practical realization of SCA structures. Efforts in this field are now very broadly based, with about 15 laboratories either constructing or seriously proposing devices using superconducting rf structures. There have been two excellent summary papers in the field within the last two years. The more recent one, by P. B. Wilson, ${ }^{4)}$ discusses the $\therefore$ world-wide status of superconducting accelerator technology. The earlier paper, by J. P. Turneaure, ${ }^{5)}$ reviews the more general area of rf superconducting applications (not merely accelerators), and summarizes fabrication and processing techniques now employed in the production of superconducting structures for high field gradient applications. It is not appropriate, here, to enter into a detailed summary of SCA programs. I shall, rather, discuss certain aspects of the Stanford SCA program in order to illuminate the subject and problem areas.

\subsection{Manufacture and Assembly of Superconducting Cavities}

Superconducting cavities have been fabricated from a number of different superconductors. The early work, as mentioned above, employed lead-plated, copper structures. More recently, interest has focussed on $\mathrm{Nb}$, particularly for high field $\mathrm{rf}$ devices. At HEPL, 20-cm diameter $\mathrm{Nb}$ cavities resonant at $1300 \mathrm{MHz}$ are being manufactured for the SCA. These cavities are of such a diameter that machining a large number from solid $\mathrm{Nb}$ would be costly, in terms of both material and machining time. Instead, $5-\mathrm{mm}$ thick $\mathrm{Nb}$ sheet is hydroformed into cups, which after stress relief at $975^{\circ} \mathrm{C}$, are machined to final dimensions and electron beam (EB) welded together to form the final cavity. Additional longitudinal rigidity is obtained by EB welding square $\mathrm{Nb}$ bars to the exterior cavity surfaces. A $1-\mathrm{m}$ long fabricated section is shown in Fig. 7. These $1-\mathrm{m}$ sections are then chemically cleaned, fired at $1900^{\circ} \mathrm{C}$ 
in a $10^{-8}$ to $10^{-9}$ Torr vacuum for a few hours, chemically polished $(\sim 100 \mu \mathrm{m})$, and fired under ultrahigh vacuum a second time at $1900^{\circ} \mathrm{C}$. It is important to avoid any contamination during the later stages of processing. Impurities present during the last firing diffuse into the surface. Particles introduced subsequent to processing provide high field sites for cavity degradation. After processing, seven 1-m cavity structures are joined by indium seals to form the 6-m long structure shown enclosed in its dewar in Fig. 8.

\subsection{Installation and Operation}

A photograph of the Stanford injector system installed in its tunnel is shown in Fig. 9, and the system is schematically diagrammed in Fig. 10. The injector dewar and the first 6.2-meter accelerating section dewar are easily visible, each downstream of a turbomolecular pump which stands in the aisle. In operation, the dewars are full of liquid helium at a temperature near $1.8^{\circ} \mathrm{K}$, below the $\lambda$-point. In this temperature region, the excellent heat transfer properties of the superfluid prevent temperature gradients from occuring in the fluid, although the Kapitza resistance at the metal-fluid interface must of course be reckoned with.

It is of considerable interest to report first the function and results of tests $^{6)}$ on the injector system, and then the latest available results of tests on the injector and first two 6.2-m accelerator sections, which are underway as this paper is being written (December 1973).

Referring to Fig. 10, 100-kV electrons from the gun are chopped, bunched, and injected into the capture section, in which they are further bunched and accelerated to an energy of $1.8 \mathrm{MeV}$. These electrons are brought to an energy of $8 \mathrm{MeV}$ in the pre-accelerator section. The $8-\mathrm{MeV}$ beam passes through the beam filter, a transport system consisting of four bending magnets, .. with several solenoidal lenses and adjustable slits. (The beam filter has three main functions: 1) It removes low-energy electrons, such as those formed by 
field emission in the capture or pre-accelerator sections. 2) It permits additional phase bunching to be achieved by varying the path length as a function of energy. 3) Deflection and displacement of the injector beam is provided, in order to allow reinsertion into the main accelerator of a highenergy recirculated beam, as will be mentioned later.) The injector beam analysis was performed with the first $6.2-\mathrm{m}$ superconducting accelerator section used to measure the spread in phase of the beam, while the magnetic spectrometer analyzes the beam energy. The beam quality of a long electron linear accelerator depends principally on the phase-space area of the beam as it emerges from the injector. For the HEPL injector, the longitudinal phase.space area $\Delta \mathrm{E} \cdot \Delta \theta$ of less than $12 \mathrm{keV} \cdot \operatorname{degree}(\mathrm{FWHM})$ at $8 \mathrm{MeV}$ was measured for currents up to $250 \mu \mathrm{A}$. By using property (2) of the beam filter, a phase spread $\Delta \theta$ of less than one degree could be obtained. The transverse phasespace area of the injector was less than $\pi \mathrm{mm} \cdot \mathrm{mrad}$ at $8 \mathrm{MeV}$. The longitudinal phase-space area indicates that an energy resolution of $100 \mathrm{ppm}$ can be achieved at energies of $200 \mathrm{MeV}$ and higher. In these injector tests, the quality and stability of the beam was measured. At $8 \mathrm{MeV}$ the energy fluctuated less than 30 ppm over a 30 -minute period. Increasing the current from 10 to $250 \mu \mathrm{A}$ (by changing only the gun emission) caused an energy shift of only $60 \mathrm{ppm}$.

Measurements on $6.2-\mathrm{m}$ accelerating section No. 1 have just been completed at this writing (December 1973). For continuous wave operation, an accelerating gradient of $2.62 \mathrm{MeV} / \mathrm{m}$ with $Q>9 \times 10^{9}$ was measured. Under pulsed operations, at $50 \%$ duty factor and $10 \%$ duty factor, gradients of $3.0-$ and $3.75-\mathrm{MeV} / \mathrm{m}$ respectively with $\mathrm{Q}$ values of $>5 \times 10^{9}$ were obtained. The theoretical $Q$ under these conditions is $2 \times 10^{10}$, and it may have been achieved. The values measured are limited by inability to measure a change of less than 5 watts dissipated in the liquid helium. High $Q^{\prime} \mathrm{s}$ have been observed in $6.2-\mathrm{m}$ 
section No. 2, but tests are not yet complete on this unit. Previous tests have accelerated a $500 \mu \mathrm{A}$ electron beam through a $6.2-\mathrm{m}$ section. A severe limitation on the maximum current of long linear accelerators is beam break up (BBU), which is caused by excitation by the beam of cavity modes which deflect the following electrons in the beam out of the accelerator. In the 55cavity sections described here, there are 275 BBU modes. In the HEPL SCA all of these have been damped by probes which absorb energy at the BBU frequency, but not at the $1300 \mathrm{MHz}$ fundamental. In fact, the isolation to the fundamental is so high $\left(\sim 10^{6}\right)$ that the probes are allowed to dissipate all absorbed energy in the helium bath.

\subsection{Future Plans at Stanford}

A recirculating linear accelerator system has been proposed, which would employ eight $6.2-\mathrm{m}$ accelerating sections: With four recirculations (five passes through the linac) a maximum energy of $700 \mathrm{MeV}$ is attainable at $50 \%$ duty factor, with the gradients measured above. A prototype of this system, using only four $6.2-\mathrm{m}$ sections, is shown in Fig. 11. All four sections are already built, two are installed in the tunnel and are presently under test, as described above.

\subsection{Outstanding Problems and Research Areas}

The theoretically achievable accelerating gradient for $\mathrm{Nb}$ structures is about $50 \mathrm{MeV} / \mathrm{m}$. At present, $1300 \mathrm{MHz}$ multi-cavity structures can achieve gradients, as reported, of $3.75 \mathrm{MeV} / \mathrm{m}$ at $10 \%$ duty factor. Cavities resonant at $2800 \mathrm{MHz}$ show about 1.7 times the gradient of $1300 \mathrm{MHz}$ cavities ${ }^{10)}$. What is the cause of this limitation ? Electron loading is the principal factor limiting the maximum field in SCA cavities resonant below about $2 \mathrm{GHz} .{ }^{7,8,9)}$ These electrons are field emitted from regions of the cavity exhibiting high electric fields, caused by microscopic surface anomalies which locally enhance the electric field. Electron loading has been investigated by observing $x$-radiation 
as a function of various cavity characteristics. ${ }^{10)}$ It appears that the electric field enhancement factor $\beta$ and the consequent $x$-radiation level depend on the cavity vacuum, being generally less for permanently evacuated cavities than for continuously pumped cavities with parts of the vacuum system at room temperature. The $\mathrm{x}$-radiation data for cavities resonant at 2800 and $1300 \mathrm{MHz}$ are consistent with a statistical model for high $\beta$, field-emitting projections, giving appropriately higher emission from the larger, lower frequency cavities. There is a considerable body of evidence which is consistent with the existence, at low temperatures, of field emission enhancement by surface states ${ }^{11}$ ) produced by gases adsorbed on the metal surface and subsequently bombarded by electrons and $\mathrm{x}$-radiation. The Fowler-Nordheim equation for field-emission can be modified to include the effects of electron dynamics in rf fields, $x-$ radiation production, and absorption of $x$-radiation by the cavity walls. The modified equation for the external x-radiation intensity $\dot{N}$ is: $\left.{ }^{11}\right)$

$$
\dot{N}=C(\beta)^{5 / 2} E^{7.5} \exp \frac{-6.83 \times 10^{7} v(y) \phi^{3 / 2}}{\beta E},
$$

where $\mathrm{C}$ is proportional to the field-emitting area, $\mathrm{E}$ is the macroscopic electric field at the metal surface in $\mathrm{V} / \mathrm{cm}, \phi$ is the work function in $\mathrm{eV}$, and $\mathrm{v}(\mathrm{y})$ is a well-known, tabulated ${ }^{12)}$ function of $\mathrm{E}^{1 / 2} \phi^{-1}$.

Following a suggestion of the author, and subsequent research at the Stanford Linear Accelerator Center on room-temperature d.c. sputtering with helium, which was demonstrated to significantly reduced field emission, the HEPL group recently showed that the field emission properties of $\mathrm{Nb}$ cavities can be improved by selective sputtering with helium gas at low temperatures. A specific low-temperature surface state effect was also observed. Pure helium gas was admitted to a pressure of $10^{-4}$ Torr (measured at room temperature), until avalanche ionization was observed in the superconducting 
cavity. The pressure was then reduced to below the avalanche level and helium-ion sputter processing began. The field level in the cavity was increased as required to maintain a moderate radiation level $(10-100 \mathrm{mR} / \mathrm{h})$ at a distance of $1 \mathrm{~m}$ from the cavity. During processing, the radiation level fluctuated, but the general radiation level progressively decreased for any given field level. Results of such an experiment are shown in Fig. 12 for an unprocessed cavity, and after $5,9,23$, and 51 hours of processing. The measured $x$-radiation values are fitted to the modified Fowler-Nordheim equation. Using the rf power absorbed by the cavity, the average energy gained from the cavity rf fields by an electron, and the time-averaged field emission current density, a value for the effective emitting area can be obtained. The estimated upper bound on the emitting area obtained in this way at the end of processing is $1.5 \times 10^{-9} \mathrm{~cm}^{2}$. Since emission areas typically observed for single projections are $\gtrsim 3 \times 10^{-11} \mathrm{~cm}^{2}$, thexe are less than 50 emitting sites. From analysis of the plots in Fig. 12, an upper bound on the emitting area before processing can be made, and is $3 \times 10^{-13} \mathrm{~cm}^{2}$. This latter value is not consistent with the typically observed area of $3 \times 10^{-11} \mathrm{~cm}^{2}$, but is consistent with the interpretation that initially the field emission is dominated by resonant tunneling through surface states. ${ }^{11)}$. Helium-ion sputter processing quickly removes the adsorbed atoms responsible for resonant tunneling, after which the field emission can be properly described by the Fowler-Nordheim theory. This interpretation allows for the large area increase, since the apparent emitting area associated with resonant tunneling can be as small as a single adsorbed atom. ${ }^{13)}$ During the later phases of processing, many sites contribute to the emission, and sputtering of the $\mathrm{Nb}$ reduces the values of $\beta$, as was observed in the room temperature sputtering experiments.

A furnace has been constructed at HEPL, and a high temperature rf window has been developed, which allow final high-temperatures processing of the cavity, and pinch-off of the complete unit ready for cryogenic rf testing, 
eliminating any gas admission. This research tool should prove very powerful in investigating the resonant surface state behaviour, and point the way toward possible improvements in attainable energy gradients.

\section{Acknowledgments}

The author would like to thank Professors Alan Schwettman and John Turneaure for their very kind cooperation during the writing of this paper, as well as in years past. 


\section{REFERENCES}

1) R. B. Neal, J. Vac. Sci. Technol. 2 (1965) 149.

2) R. B. Neal, Physics Today 20 (1967) 27.

3) E. L. Garwin and E. W. Hoyt, "Hot Lithium Condensation on Room Temperature Copper", SLAC TN 73-2 (1973) p. 82.

4) P. B. Wilson, "Current Status of Superconducting Accelerator Technology", Proc. 1972 Proton Linear Accelerator Conf. (Los Alamos Scientific Laboratory, LA5115, 1972) p. 82.

5) J. P. Turneaure, "Status of Superconductivity for rf Applications", p.621, Proc. 1972 Applied Superconductivity Conf. in Annapolis Md, IEEE Pub. 72CH0682-5-TABSC (1972).

6) L. R. Suelzle, "Progress on rf Electron Superconducting Accelerators", presented at the 1973 Particle Accelerator Conf., San Francisco, Calif.

7) J. P. Turneaure and N. T. Viet, Appl. Phys. Letters 16 (1970) 333.

8) J. P. Turneaure, IEEE Trans. Nucl. Sci. NS-18 (1971) 166.

9) C. Lyneis, M. Mc Ashan and N. T. Viet, Proc. 1972 Proton Linear Accelerator Conf. (Los Alamos Scientific Laboratory, LA5115, 1972) p. 98 .

10) C. Lyneis, Y. Kojima, J. P. Turneaure and N. T. Viet, "Electronloading in L- and S- Band Superconducting Niobium Cavities", presented at the 1973 Particle Accelerator Conference, San Francisco, Calif.

11) H. A. Schwettmann, J. P. Turneaure and F. F. Waites, J. Appl. Phys., to be published.

12) J. W. Gadzuk, Phys. Rev. B 1 (1970) 2110.

13) E. W. Plummer and R. D. Young, Phys. Rev. B 1 (1970) 2088. 
Figure 1 Cross section of linear accelerator housing and klystron gallery.

Figure 2 Disk-loaded waveguide configuration.

Figure $3 \quad 12.2-\mathrm{m}$ support and alignment girder assembly, consisting of four $3-\mathrm{m}$ accelerator sections on a $61-\mathrm{cm}$ support.

Figure 4 View looking east along the tunnel of the $3.2-\mathrm{km}$ accelerator.

$\therefore \quad$ Figure $5 \quad$ Main vacuum system for each $102-\mathrm{m}$ sector.

Figure 6 Installation schematic for the $\mathrm{Li}^{6}$ polarized electron source (PEGGY).

Figure $7 \mathrm{Nb}$ accelerator structure for HEPL superconducting linear electron accelerator. This structure is $1-\mathrm{m}$ long.

Figure 8 Assembled 6.1-m accelerator dewar module.

Figure 9 The HEPL injector system and first accelerating section dewar installed in the tunnel.

Figure 10 Schematic diagram of HEPL superconducting injector and beam analysis system.

Figure 11 Schematic layout of prototype recirculation system.

Figure 12 Modified Fowler-Nordheim plots of $\mathrm{x}$-radiation data for various helium-ion sputter-processing times. The selective helium-ion sputter-processing times and enhancement factors are (a) no processing and $\beta=547$, (b) 5 hours and $\beta=353$, (c) 9 hours and $\beta=236$, (d) 23 hours and $\beta=215$, and (e) 51 hours and $\beta=206$. 